\title{
$\nabla$ Ocular Camouflage Adoption for an Anophthalmic Anthrophobic Patient
}

\section{IJCRR \\ Section: Healthcare \\ ISI Impact Factor \\ (2019-20): 1.628 \\ IC Value (2019): 90.81 \\ $\operatorname{SJIF}(2020)=7.893$}

\section{Vini Rajeev', Ajay Jain'2, Rajeev Arunachalam', Sridevi Ugrappa ${ }^{1}$}

'Senior Lecturer, Faculty of Dentistry, Asian lnstitute of Medicine, Science and Technology (AIMST) University, Malaysia; ${ }^{2}$ Associate Professor,

Faculty of Dentistry, Asian Institute of Medicine, Science and Technology (AIMST) University, Malaysia.

(ब) (1) 8

Copyright@IJCRR

\section{ABSTRACT}

Introduction: An ideal ocular prosthesis that imitates a natural eye can make an individual improve their social relationships and can bring back a stress-free environment thereby advancing the quality of life.

Objective: Loss of an eye can affect an individual psychologically and can prevent them from leading a normal social life. The present case report focuses on the steps in the fabrication of a pragmatic custom ocular prosthesis that eliminated the social phobia faced by a young adult.

Case Report: A 20-year-old female patient requested the rehabilitation of her anophthalmic socket resulted from surgery due to a tumour. Even though she was using a stock ocular prosthesis, the reduced dimension of the palpebral fissure resulted in poor retention of her existing prosthesis thereby causing frequent displacement. Of the range of eye prosthesis available, a custom-made ocular prosthesis was suggested to simulate a natural eye thereby increasing the confidence and self-esteem of an affected person.

Discussion: Various techniques have been utilized over the years in the fabrication of custom ocular prosthesis. The life-like camouflage of the natural eye can be obtained by replicating the colour shape and size of the normal eye. Crafting a realisticlooking artificial eye is a state of art and improves the cosmetic effect of the face. The prosthesis was made of high-quality acrylic material accurately and was hand-painted to match the colour of the natural eye.

Conclusion: The surgical removal of the eye is a disheartening situation and the receptivity of the society on this aspect cannot be predicted. In this situation, a well-fabricated prosthesis can conceal the disfigurement and improve the patient's social interaction.

Key Words: Anophthalmic socket, Artificial eye, Custom eye prosthesis, Enucleation, Ocular prosthesis, Scleral shell

\section{INTRODUCTION}

An anophthalmic socket is most undesirable and might affect the psychosocial aspects of the affected individual. Such individuals face emotional and behavioural changes and their quality of life will be affected if they remain with their compromised facial integrity. An ideal ocular prosthesis that imitates a natural eye can make an individual improve their social relationships and can bring back a stress-free environment thereby advancing the quality of life. The relic of an ocular prosthesis dates back to as early as 2800 years ago and the sixteenth century made a dramatic change with the introduction of ocular prosthesis made of metals like copper, bronze, and gold in the contracted anophthalmic socket. The fabrication of 'Hypoblepharae' by Ambrose Pare in the sixteenth century changed the future and quality of life of those suffering from loss of an eye. The attempts of the pioneer in ocular prosthesis started with gold and silver, but he later improved the design using glass and porcelain, which set a zenith in the field of maxillofacial prosthodontics. ${ }^{1}$ Glassprosthesis, was the popular material from the $17^{\text {th }}$ century until the introduction of synthetic materials in the twentieth century. Currently, ocular prostheses can be fabricated using cryolite glass and acrylic resin. The silicone material also plays a major role in the rehabilitation of orbital prosthesis to cover the deformations in the periorbital facial region. ${ }^{2}$ Most recently, the prominence of computer-aided design and computer-aided manufacturing and rapid prototyping has ac-

\section{Corresponding Author:}

Dr. Vini Rajeev, Senior Lecturer, Department of Prosthodontics, Faculty of Dentistry, AIMST University, Jalan Bedong Semeling, Kedah, Darul Aman, Malaysia 08100; Phone: +60143928025; E-mail: vinirajeev09@gmail.com

ISSN: 2231-2196 (Print)

Received: 02.10 .2020
ISSN: 0975-5241 (Online)

Revised: 22.11 .2020
Accepted: 14.01 .2021
Published: 19.05 .2021 
tivated the era of high-speed prosthodontics thereby promoting the fabrication of $3 \mathrm{D}$ printed customized prostheses.

Ocular defects can be congenital or acquired. Acquired ocular defect ensue by three types of surgery (a) Evisceration, the removal of the internal content of ocular bulb keeping the bulb in the orbit (b) Enucleation, the removal of whole ocular bulb keeping the adjacent structures in the orbit; and (c) Exenteration, the removal of the whole content, eyelid, and posterior coverage of epidermal tissue with a graft. These defects should be considered for rehabilitation to improve the esthetic appearance and functional requirements that include preventing eyelid deformation, maintaining adequate width of the orbital fissure, protecting the cavity and orienting the lacrimal flux thereby avoiding its accumulation. ${ }^{2}$

Modugno et al. in their research among 8018 ocular prosthesis wearers, found that $63 \%$ were males and $37 \%$ were females, with a mean age of 29 years. The main cause of ocular prosthesis application was a traumatic event (54\%). Based on the above study, there was no significant difference in gender on other most common causes for ocular rehabilitation which

includes end-stage ocular diseases, tumours, and congenital malformations. ${ }^{3}$ Based on another research conducted in the southern zone of India on 118 individuals over 5 years, $66 \%$ of the male population had ocular defects. Trauma (46\%) and pathogenic $(44 \%)$ causes were the main reasons over nonspecific $(8 \%)$ and congenital $(2 \%)$ reasons. ${ }^{4}$ Goiato et al. stated that ocular prosthesis causes a positive influence on the patients' socio-psychological status. ${ }^{5}$

\section{CASE REPORT}

A 20-year-old female patient reported to the Faculty of Dentistry, AIMST University for the rehabilitation of her anophthalmic socket. Her medical history revealed enucleation of the left eye due to retinoblastoma at the age of 3 years. The patient used a conformer immediately after surgery and discontinued its use within two weeks due to discomfort. The patient started wearing a stock ocular prosthesis only seven years after the surgery which has led to the reduction in the space of the palpebral fissure. The lack of space resulted in poor retention of the prosthesis thereby causing frequent displacement of the prosthesis. Overall, the patient was dissatisfied with the esthetics and the fit of the prosthesis. This case report aims to display the fabrication processes of an ocular prosthesis which improved the confidence and attitude of a twenty-year-old university student.

\section{Patient evaluation}

The patient has changed her prefabricated ocular prosthesis multiple times and the current prosthesis was being used for
1 year. Examination of the socket revealed no signs of inflammation. The patient's stock eye did not correspond with the pupil position of the right eye (Figure 1). Preoperative evaluation of the socket was performed and the patient was explained about the possible limitations of the treatment.

\section{Observations during clinical examination}

- Evaluation of the muscular control of the palpebra was performed and was observed as minimal compared to the right eye. The movement was better during opening and closing compared to lateral movements.

- The palpebral fissure dimension was reduced. Internal anatomy of the socket in a resting position and during full excursive movements of the eye musculature revealed a lack of depth for the eye bed increasing the challenge of placement of the prosthesis. The surgical scar tissue at the outer canthus of the left eye made the area very shallow compromising in retention of the prosthesis. The anatomy near the medial canthus was the only favourable area for retention of the prosthesis.

- Mobility of the posterior wall of the defect during the movement of the intact eye was absently necessitating the use of spectacles to mask the discrepancy during lateral movement

\section{Impression}

The ophthalmic socket was recorded using light body addition silicone impression material (Aquasil Ultra LV, Dentsply). A modified impression technique adapted from the concepts of Allen and Webster was used for this patient. ${ }^{6}$ The patient's previous custom made acrylic conformer was modified with wax and duplicated using acrylic resin to use as an impression tray. The conformer was perforated to a 5 $\mathrm{mm}$ diameter hole at the approximate location of the pupil. Multiple minute perforations were made over the remaining surface. An acrylic hollow cylinder of $5 \mathrm{~mm}$ diameter and $5 \mathrm{~mm}$ length was fabricated and attached to the tray (Figure 2a). The tray was inserted into the socket and the mixing tip for addition silicone was inserted into the cylinder and the impression material was dispensed through it till it filled the socket and excess material flown out through the additional holes. The patient was seated erect gazing at a spot in front until the impression procedure was completed to ensure the recording of the approximate position of the eye and the muscles (Figure 2b).

\section{Fabrication of working cast}

After the setting of the impression material, the tray was removed from the socket. A two-piece Type IV Die stone cast was poured using a plastic dish and the lower part of the impression was immersed initially. Once the die stone was set, the separating medium was applied to the surface. Index notches were prepared on all four sides for reorientation and the second layer was then poured (Figure 2c). 
Fabrication and try-in of the sclera wax pattern

Molten modelling wax was poured into the aligned two-piece cast. The wax pattern was contoured and carved to fit into the anophthalmic socket. Try-in of the wax pattern was done to check for the retention and support, size, unobstructed eye movement, and eyelid competency while closing.

\section{Positioning of Iris on the scleral pattern}

The custom iris disc with the stalk was fabricated using a metal mould and clear acrylic. The patient was instructed to fix the gaze to an object kept 3 feet in front and at eye level. Digital vernier calliper measured $11.11 \mathrm{~mm}$ as the diameter of the right iris. The fabricated iris disc was trimmed $0.5 \mathrm{~mm}$ less than the right iris to accommodate the limbus at the final stage of iris painting. The position of the iris of the natural eye about the outer canthus, inner canthus and facial midline was transferred to the left side for the positioning of the left iris. The position of the iris about upper and lower lids was also measured and transferred to the affected side using the calliper (Figure 3).

\section{Iris disc painting and attaching of iris lens}

Iris painting was performed using oil paints (Camel Artists'Oil Colour). The iris painting was done in five steps to simulate the pupil, the limbus, the collarette, the stroma and the individual striations. The black base colour was given to the iris according to the hue of the natural iris. The pupil was painted first in the centre of the iris disk. Additional colour like flake white, titanium blue, yellow ochre and burnt sienna was used to create the remaining features within the iris. Very fine striations were produced on the disc using a camel hairbrush. The paints mixed with oil were more intense than the colour of the natural iris to avoid fading after processing. The colour was compared with the natural iris by applying a droplet of water to the centre of the disc. The water allows the iris characterization to be viewed as it will appear within the iris-corneal assembly. Once the colour matching was perfect, the prefabricated iris button was attached to the corneal disc using Monopoly syrup and one drop of cyanoacrylate. The stalk of the iris disc was trimmed off and the assembly was attached to the wax pattern for further verification.

\section{Acrylisation and characterization of sclera}

Final try in of wax pattern with the iris-lens assembly was performed. The esthetics and function were compared to the right eye. The patient was advised to perform the eye movements in all direction (Figure 4a-f). On confirmation of the effectiveness of the wax pattern, it was flasked and packing was performed using A2 shade heat cure acrylic resin for obtaining the sclera. A short curing cycle was carried out. After acrylisation, $1 \mathrm{~mm}$ of the scleral acrylic was reduced to aid for further characterization.
Oil paints of cobalt blue, yellow ochre and burnt sienna were used for colouring. Red cotton fibres resembling nerves were attached using monopoly syrup for enhancing the natural appearance of the prosthesis in comparison with the right eye. The characterization on the outer layer was secured by acrylising the remaining $1 \mathrm{~mm}$ space with clear heat cure acrylic(Figure 5). The final trimming and polishing were done and the prosthesis was inserted (Figure 6a-b).

\section{Post insertion instructions}

The patient was demonstrated on the method of insertion and removal and was advised to remove it every night. The patient was advised to clean the prosthesis once every month using a mild soap solution without alcohol content to clean it. Use of eye lubricant solution was also advised to avoid drying of the eye bed and clearing of discharges inside the socket. The patient was asked to return to the operatory for further trimming and polishing if she experiences any pain while using the prosthesis.

\section{DISCUSSION}

Anthropophobia, a major concern for individuals with surgical ocular defects can be treated with an ocular prosthesis, an artificial replacement of the bulb of the eye. A multidisciplinary approach involving ophthalmologist, oral and maxillofacial surgeon, plastic surgeon, and maxillofacial prosthodontist is essential to impart successful rehabilitation and maintenance care to restore the patient's quality of life.

Various techniques in the fabrication of ocular prosthesis have been discussed in the literature. Ocularists and prosthodontist always attempt newer impression and processing techniques required in the fabrication of eye prosthesis. ${ }^{7-10}$ As a result, prefabricated and custom made ocular prosthesis are in demand. Implant-supported ocular prosthesis is also in call nowadays. Even though ocular implant enhances the coordinated movement of the affected eye to an extent, due to the associated economic factors; many individuals have to decline the option. This situation favours the benefit of custom made ocular prosthesis which superior in esthetic and functional outcome in comparison with prefabricated prosthesis.

The expertise of an operator in providing a better quality of life for a patient with the enucleated eye can be enhanced by empirically fitting a prefabricated eye, modifying a stock eye by making an impression of the ocular defect or by providing a custom made eye prosthesis to fill the socket. Based on the studies by Beumer et al., the ocular prosthesis should remain in contact with the tissue bed thereby distributing the pressure within the socket evenly. ${ }^{11}$ Use of a prefabricated prosthesis can compromise on this requirement and hence should be avoided to the maximum. The socket constantly produces mucus and secretions and the prosthesis to an extent should be able to selfcleanse these secretions. The unfitted areas of the prefabricated 
prosthesis can act as stagnation zones thereby irritating the mucosa and emerge as a potential source of infection. ${ }^{11,12}$ Considering the condition of the socket, poor fit of the existing stock eye and potential side effects of the prefabricated prosthesis, a custom ocular prosthesis was suggested for this patient.

The brilliance of an eye prosthetist is revealed in the characterization of the iris. This determines the esthetic acceptance of prosthesis by the patient and the society. Various techniques can be adapted for iris colouring, selection and positioning. ${ }^{13}$ Various techniques in iris characterization has been discussed in the literature. ${ }^{14,15}$ Prefabricated iris discs can be selected and modified, painting of custom made iris discs can be done or even digital imaging and printing of iris can also be done. In this case, painting of the iris has been performed using oil paints.

Based on the studies conducted by Fernandes, all paints underwent exhibited ageing whereas; oil paint had the highest resistance to accelerated aging. ${ }^{16} \mathrm{Hence}$, oil paints were used for the characterization in this case. The monopoly syrup attributed to better handling and fixing of colours. Red cotton fibres concluded the final characteristic feature of the sclera.

Post insertion care plays a major role in maintaining a flawless prosthesis. The prosthesis should be cleaned whenever there is clogging of precipitate inside the socket. This ensures the removal of discharges and enabled the conjunctiva to increase lubrication. This can be done every month or a period not exceeding six months. Constant removal was contraindicated as it can cause mechanical irritation due to the friction produced during removal. Cleaning of fingers is recommended to avoid the incorporation of external dust and bacterial load on to the prosthesis. The prosthesis should be stored in water or contact lens solution after removal. In general, the prosthesis should be kept in a socket as long as it is comfortable. ${ }^{17}$ Use of spectacles will protect the natural eye and the artificial eye from external irritants.

\section{CONCLUSION}

The custom ocular prosthesis in this case was alluring and ensured maximum functionality within the anatomic limitations of the anophthalmic socket. It could exhibit various eye movements and could retain the shape of the defective socket to an extent by preventing ptosis. It could simulate the palpebral opening almost similar to the natural eye. The gaze and the colour mimicked her right eye and this artistic and innovative approach has boosted her confidence and was socio-psychologically uplifted.

\section{ACKNOWLEDGEMENT}

Authors acknowledge Ms See Gaik Lan for her contribution to the laboratory procedures involved and also AIMST University, Faculty of Dentistry for providing the facilities.

\section{Source of Funding: Self-Funded}

\section{Conflict of Interest: NIL}

Authors' Contribution: Dr. Vini Rajeev - Clinical procedures and manuscript preparation

Dr. Ajay Jain - Clinical Procedures

Dr. Rajeev Arunachalam - Manuscript editing

Dr. Sridevi Ugrappa - Manuscript editing

\section{REFERENCES}

1. Martin O, Clodius L. The history of the artificial eye. Ann Plast Surg 1979;3(2):168-71.

2. Goiato MC, de Caxias FP, Santos DM. Quality of life living with ocular prosthesis. Expert Rev Ophthalm 2018;13(4):187-189.

3. Modugno A, Mantelli F, Sposato S, Moretti C, Lambiase A, Bonini S. Ocular prostheses in the last century: a retrospective analysis of 8018 patients. Eye (Lond) 2013;27(7):865-870.

4. Raj N, Singh M, Raj V, Anwar M, Kumar L. Prevalence of ocular defects among patients visiting in an institutionalized hospital setting: A cross-sectional study. Natl J Maxillofac Surg 2016;7(1):67-70.

5. Goiato MC, dos Santos DM, Bannwart LC, et al. Psychosocial impact on anophthalmic patients wearing an ocular prosthesis. Int J Oral Maxillofac Surg 2013;42(1):113-119.

6. Allen L, Webster H. Modified impression method of artificial eye fitting. Am J Ophthalmol 1969;67:189-218.

7. Bartlett S, Moore D. Ocular prosthesis: A physiologic system. J Prosthet Dent 1973;29:450-9.

8. Brown K. Fabrication of an ocular prosthesis. J Prosthet Dent 1970;24:225-35.

9. Laney WR, Gardner AF. Maxillofacial prosthetics. PSG Publishing Company, Littleton, Massachusetts; 1979.

10. Ow R, Amrith S. Ocular prosthetics: Use of a tissue conditioner material to modify a stock ocular prosthesis. J Prosthet Dent 1997;78:218-22.

11. Beumer J, Curtis TA, Firtell DN. Maxillofacial rehabilitation: Prosthodontic and surgical consideration. CV Mosby Co, St. Louis; 1979.

12. Pathak C, Pawah S, Singh G, et al. Prosthetic rehabilitation of completely blind subject with bilateral customised ocular prosthesis: a case report. J Clin Diagn Res 2017;11(1):ZD06-ZD08.

13. Taicher S, Steinberg HM, Tubiana I, et al. Modified stock eye ocular prosthesis. J Prosthet Dent 1985;54(1):95-98.

14. Lanzara R, Thakur A, Viswambaran M, Khattak M. Fabrication of ocular prosthesis with a digital customization technique - A case report. J Family Med Prim Care 2019:8(3):123942.

15. Gupta L, Aparna IN, Dhanasekar B, Prabhu N, Malla N, Agarwal P. Three-Dimensional Orientation of Iris in an Ocular Prosthesis Using a Customized Scale. J Prosthod 2014;23(3):252-255.

16. Fernandes AU, Goiato MC, Batista MA, Santos DM. Colour alteration of the paint used for iris painting in ocular prostheses. Braz Oral Res 2009;23:386-92.

17. Thakkar P, Patel JR, Sethuraman R, Nirmal N. Custom Ocular Prosthesis: A Palliative Approach. Ind J Palliat Care 2012;18(1):78-83. 


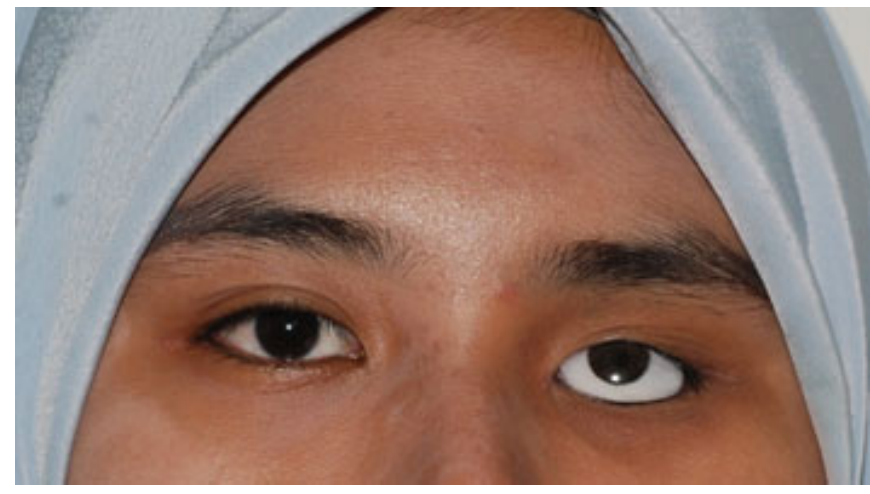

Figure 1: Preoperative view.
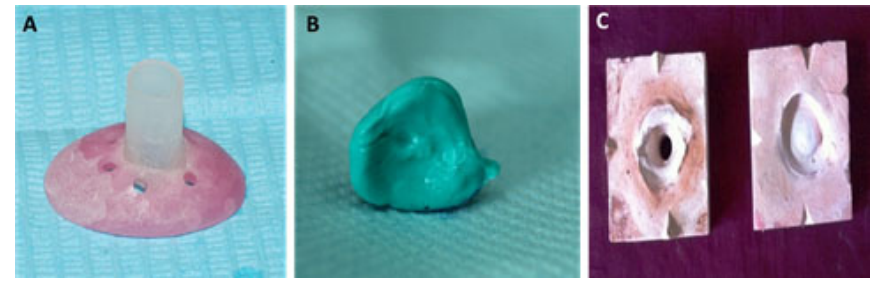

Figure 2: A. Custom impression tray, B. Ocular impression and $\mathrm{C}$. two piece split cast for wax pattern fabrication.

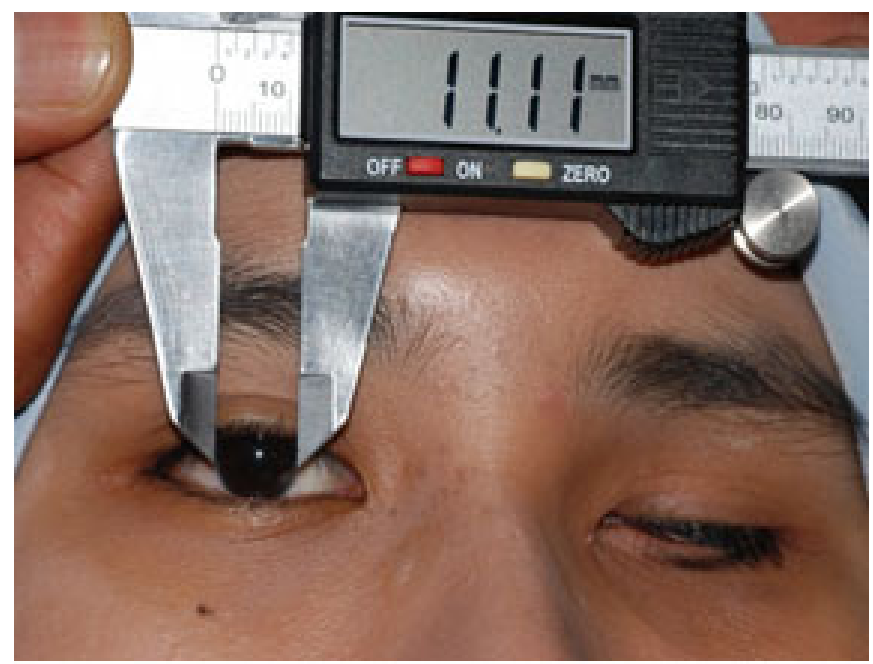

Figure 3: Evaluating the iris position.

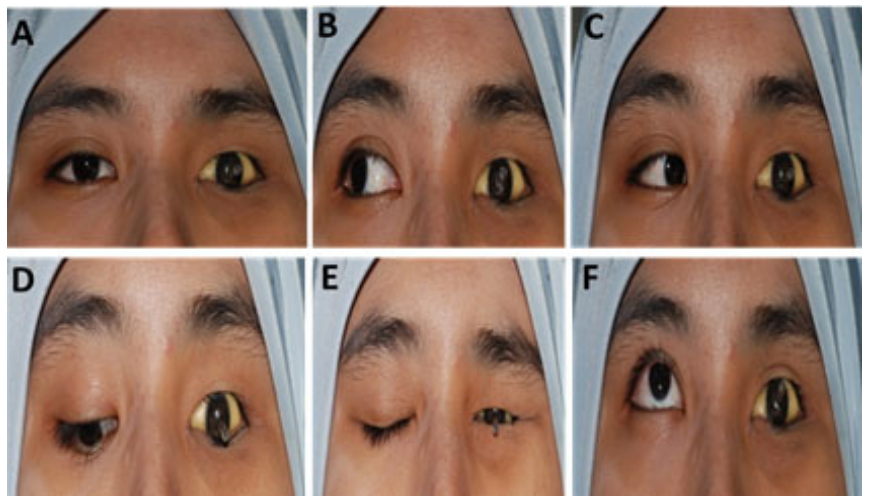

Figure 4: Evaluating the ocular movement with a wax pattern.

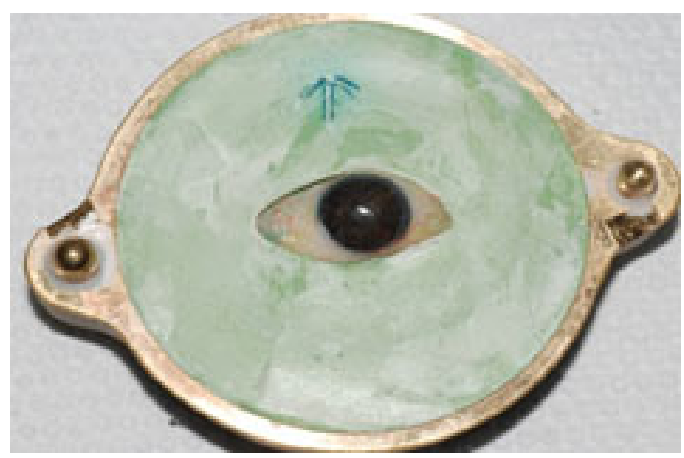

Figure 5: Final acrylisation of the prosthesis.
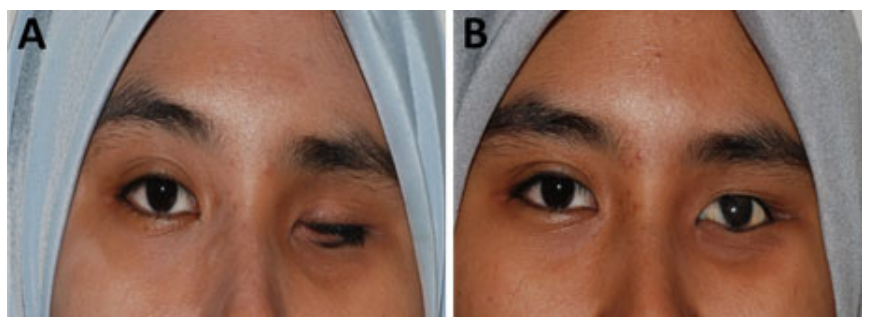

Figure 6: A. Anophthalmic socket and B. Post operative view. 